\title{
Role of 3-Dimentional Computed Tomography Scan in Video Assisted Major Pulmonary Resection in Patient with Situs Inversus Totalis
}

\author{
Adnan Raza1*, Stephen Harden², Lukacs Veres ${ }^{3}$, Khalid M. Amer'1 \\ ${ }^{1}$ Department of Thoracic Surgery, University Hospital Southampton, Southampton, UK \\ ${ }^{2}$ Department of Radiology, University Hospital Southampton, Southampton, UK \\ ${ }^{3}$ Department of Thoracic Surgery, Guy's Hospital, London, UK \\ Email: adnan raza@hotmail.com
}

Received 2 March 2015; accepted 25 July 2015; published 28 July 2015

Copyright (C) 2015 by authors and Scientific Research Publishing Inc.

This work is licensed under the Creative Commons Attribution International License (CC BY). http://creativecommons.org/licenses/by/4.0/

c) (i) Open Access

\section{Abstract}

The aim of this case report is to raise awareness about the use of 3-Dimentional Computed Tomography (3D-CT) virtual reality imaging as a routine pre-operative tool for evaluation of unusual anatomy such as Situs Inversus Totalis (SIT). We present a case of presumed lung cancer in a 58 years old lady with SIT successfully treated by VATS lobectomy via an anterior approach. She presented with an incidental solitary pulmonary nodule in her right lower lobe, which had moderate FDG uptake on the PET scan. The nodule was too deep to permit safe wedge biopsy, due to proximity of a large pulmonary arterial branch. 3D-CT images were invaluable in pre-operative assessment of the anatomy and were the key to safe completion of right VATS lower lobectomy. Sectioning the lung after retrieval of the specimen suggested a chondroid hamartoma; therefore, systemic nodal dissection (SND) was not contemplated. The final histology confirmed the diagnosis of chondroid hamartoma. In an unusual anatomy such as SIT, 3D-CT allows construction of virtual reality models that can be viewed and manipulated before and during the operation to understand the anatomy better, highlight caveats around target structures and enable localisation of lesions unlikely to be palpated intra-operatively.

\section{Keywords}

3D-CT Scan, Virtual Reality Model, Total Situs Inversus, VATS Lobectomy

\footnotetext{
${ }^{*}$ Corresponding author.
}

How to cite this paper: Raza, A., Harden, S., Veres, L. and Amer, K.M. (2015) Role of 3-Dimentional Computed Tomography Scan in Video Assisted Major Pulmonary Resection in Patient with Situs Inversus Totalis. Journal of Cancer Therapy, 6, 638641. http://dx.doi.org/10.4236/jct.2015.68070 


\section{Introduction}

Situs Inversus Totalis (SIT) is found in 1 or 2 individuals per 10,000 of the population [1]. It is of autosomal recessive inheritance, presenting with full mirror image of the normal arrangement of the thoracic and abdominal viscera, i.e., dextrocardia, right descending aorta, right position of spleen and left position of the liver. The use of video assisted thoracoscopic surgery (VATS) has increased in recent years. This was coupled by increased awareness and spatial appreciation of the intra-thoracic anatomy, to avoid iatrogenic injuries that warrant conversion to open thoracotomy. Rapid technological development and software advances of multi-detector three dimensional computed tomography (3D-CT) imaging have revolutionised the preoperative imaging of thoracic lesions and abnormalities [2]. With the 3D-CT software, a detailed virtual reality (VR) model can be reconstructed from 2D-CT, providing the surgeons with accurate conceptual image about anatomy of the lesion to avoid iatrogenic injury [3]. These VR images can be manipulated to subtract skin and bone and concentrate on special organs of interest such as airways and vascular structures as illustrated in Figure 1 and Figure 2 . They also allow 3D rotation of the VR model around an axis chosen by the user in attempt to understand surrounding anatomical relations to target structures. This allows safe planning of the operative steps, avoiding caveats and reducing the chances of conversion to thoracotomy. One of the most important caveats in unusual cases such as Situs Inversus Totalis is anatomical disorientation and it is important to disconnect the appropriate artery, vein and bronchus corresponding to a mirror image of the norm.

The aim of this case report is to create awareness of incorporating MDCT and 3D imaging as a routine preoperative imaging tool for evaluation of thoracic anatomy especially in patients with total SIT.
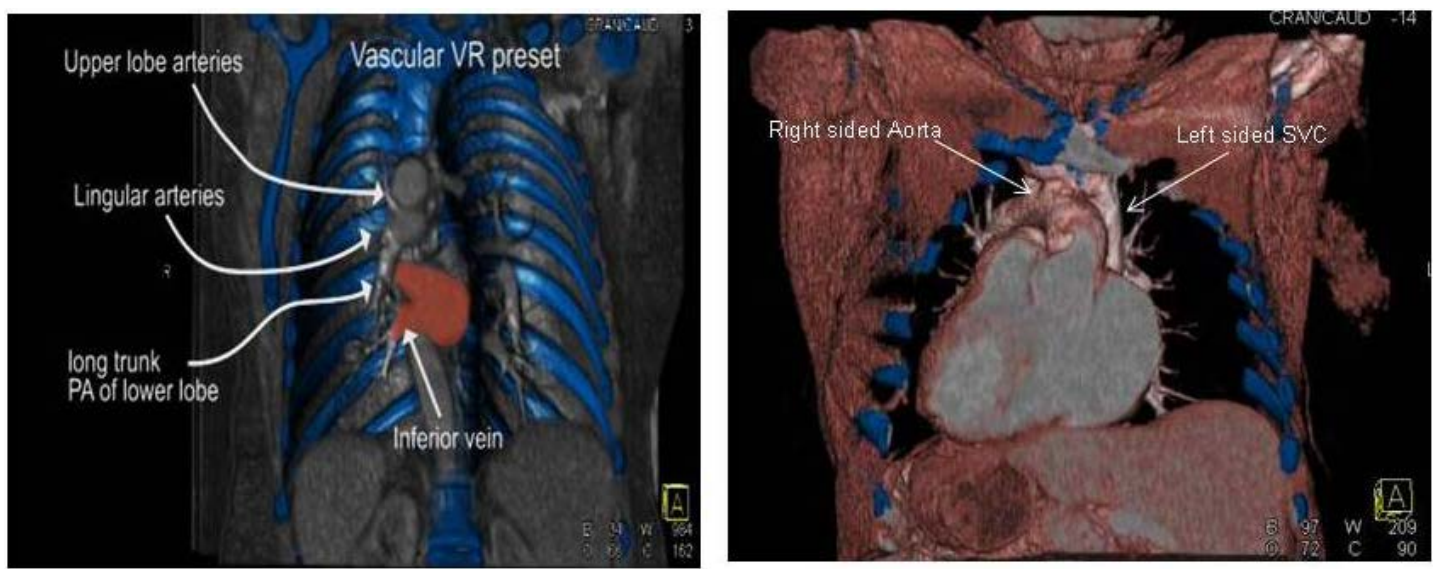

Figure 1. Manipulation of the Virtual Reality (VR) model enables scrutiny of details around important structure, such as pulmonary artery and pulmonary vein branches.
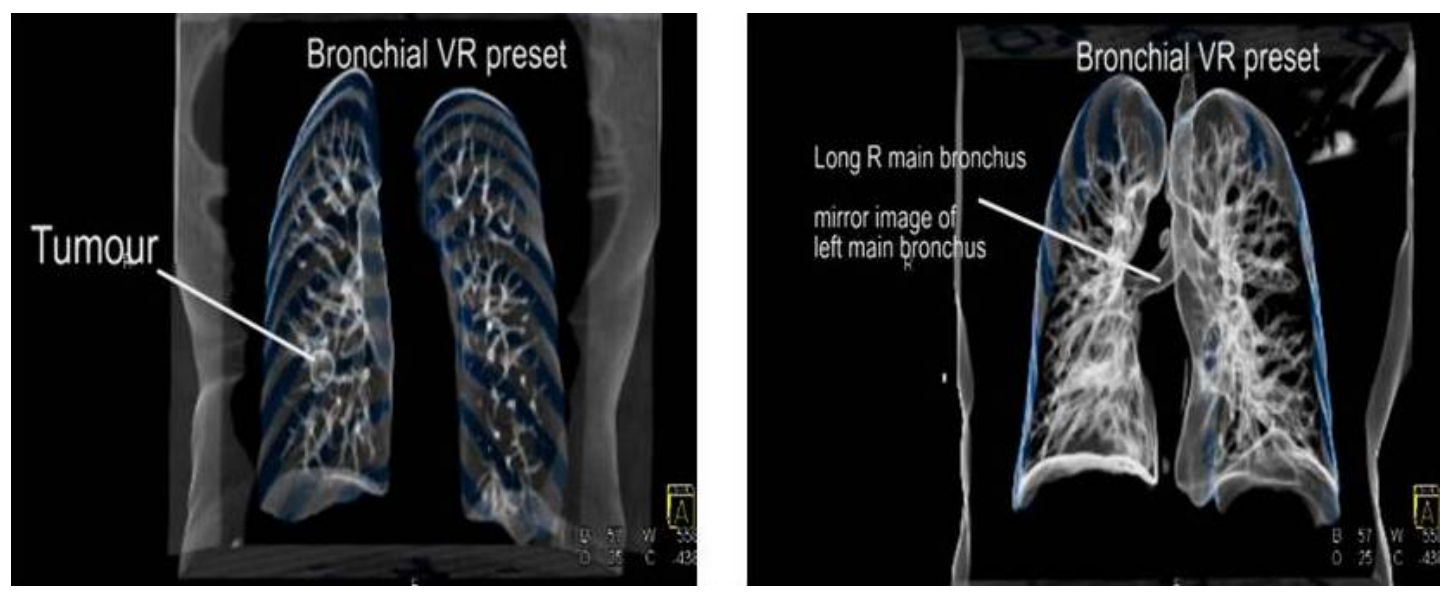

Figure 2. The bronchial preset of the VR model, showing main airways and their divisions, as well as localising the lesion within lower lobe. 


\section{Case Report}

A 58 years old lady was diagnosed with SIT 30 years prior to admission when she required mitral valve repair via median sternotomy. She presented on this occasion with progressive cough and shortness of breath. She was an active smoker. Her Chest X-ray (CXR) and Computed Tomography (CT) of the chest revealed a $2 \mathrm{~cm}$ diameter right lower lobe solitary pulmonary nodule, with dextrocardia. The nodule showed moderate flourodeoxyglucose (FDG) uptake on the Positron Emission Tomography (PET) scan. Multi-detector 3D-CT was used to reconstruct images from a 2D-CT of the chest pre-operatively and the facility to manipulate the VR model was available in the operating theatre (Figure 1 and Figure 2). Pre operative biopsy was not attempted because of the location of the nodule. Although non anatomical resection is curative for a benign lesion but in this case location of the lesion played an important role in the decision of type of surgical resection. Bronchoscopy at the beginning of surgery confirmed mirror image arrangement of bronchopulmonary segments and no endobronchial abnormality was identified.

Special precautions are required in airway management in situs inversus, as the viscus anatomy is a mirror image of the normal anatomy. For intra-operative ventilation we used a right-sided polyvinyl chloride (PVC) double lumen endotracheal tube (ETT) that was flipped upside down to reverse the bend at the teeth level. This manoeuvre helped in aligning Murphy's eye to the take-off of the left upper lobe bronchus.

Three port right VATS anterior approach procedure was initiated. On initial inspection, the arch and descending aorta were confirmed in the right chest. The right lung comprised of two lobes separated by well-defined fissure, typical of normal left sided lung. We define a nice fissure as i) the interlobar artery is clearly seen through the perivascular sleeve, ii) no crossing veins, iii) no stuck nodes on the artery and iv) peels easily by the double peanut pledget technique described by W. Walker [4]. Within the lower lobe the target nodule was identified by finger palpation. The inferior pulmonary ligament was divided and the venous anatomy ascertained. Within the fissure the interlobar artery was skeletonised and arterial anatomy ascertained. The fissure was divided by using diathermy. The bronchial anatomy was also a mirror image of a normal left side. Helped by prior knowledge from 3D-CT scan and manipulation of the VR model, the vein, artery and bronchus were disconnected respectively with confidence. On retrieval of the lobe, the lung was sectioned and the macroscopic appearances were typical of a chondroid hamartoma, therefore systematic nodal dissection was not considered. A chest drain was inserted and a paravertebral extrapleural catheter was introduced endoscopically for postoperative analgesia.

The duration of the operation was 2 hrs and 45 min with minimal blood loss of 50 mls. The recovery of the patient was uneventful and she was discharged home on 3rd post operative day. The final histology confirmed the diagnosis of chondroid hamartoma, with no malignant component. The patient was well and asymptomatic 6 month postoperatively.

\section{Discussion}

The occurrence of a solitary pulmonary nodule in the lungs of a patient with SIT is very rare. It requires particular care during surgery, as the lung anatomy is a mirror image of the normal arrangement. However, perfect mirror image arrangement cannot be presumed, even if the bronchoscopy showed a perfect mirror image bronchial pattern. Minor bronchial and/or vascular anomalies are to be assumed (5\% - 10\%) until proven otherwise [5]. Planning single lung anaesthesia in such cases is also extremely helpful with the VR model, as seen in our patient. For a right sided operation a left sided ETT is usually preferred. However, in SIT patients the early take-off of the left upper lobe might be blocked if a conventional left sided ETT was used. A bent right sided PVC ETT could be used as a left sided ETT with perfect matching of Murphy’s eye opposite the left upper lobe bronchus.

Advances in MDCT have enabled virtual reconstruction of 3D models guiding surgeons to avoid vascular or segmental airway injury with improved depth perception [2]. It provides comprehensive pre operative surgical guidance especially in patients with TSI where the vascular and segmental anatomy and its variations should be well known to surgeon prior to perform any thoracic procedure particularly VATS lobectomy. To monitor the accuracy of pre operative assessment of pulmonary vasculature, Fukuhara et al. compared the results with intra operative findings and has shown that $95.2 \%$ of pulmonary artery branches were precisely identified by pre operative 3D imaging thus safely facilitating completion of VATS lobectomy procedure [6]. Recently published data has shown efficacy of 3D reconstruction leading to improved peri-operative safety and detection of ana- 
tomical variation in patients with SIT [7] [8].

MDCT with 3D imaging has also played role in revolutionising imaging of children by improving temporal and spatial resolution, decreasing scanning time, reducing sedation rate and above all generating high quality, visually accessible and anatomically meaningful 3D images of thoracic vascular and airway structures. Familiarisation with characteristic 3D MDCT imaging appearances of various paediatric thoracic pathological and congenital anomalies can enable paediatric surgeons to develop an effective preoperative surgical plan, which in turn, can optimise surgical outcome and patient care [9].

In the not too distant future the shift from 2D to routine 3D-CT imaging and routine building of VR models will be common practice to assist in all VATS major pulmonary resections. It is foreseen that the combination of 3D-CT imaging available inside operating theatres and 3D images seen at operation to match the former, should increase safety, reduce iatrogenic errors and avoid unnecessary conversion to open thoracotomy.

\section{Conclusion}

In an unusual or complex anatomy such as SIT, 3D-CT virtual reality images help planning of anaesthesia and sequence of VATS major pulmonary resection. It confers a great deal of confidence about the structures to be disconnected and warns against caveats of surrounding structures, thus avoiding unnecessary conversion to thoracotomy.

\section{Conflict of Interest}

None declared.

\section{References}

[1] Sands, S.S. and Taylor, J.F. (2001) Prescreen Evaluation of Situs Inversus Patients. International Surgery, 86, $254-258$.

[2] McLachlan, G. (2011) From 2D to 3D: The Future of Surgery? Lancet, 378, 1368. http://dx.doi.org/10.1016/S0140-6736(11)61597-3

[3] Onuki, T. (2009) Virtual Reality in Video-Assisted Thoracoscopic Lung Segmentectomy. Kyobu Geka, 62, 733-738.

[4] Walker, W.S. (1996) Video-Assisted Thoracic Surgery: Pulmonary Lobectomy. Seminars in Laparoscopic Surgery, 3, 233-244. http://dx.doi.org/10.1177/155335069600300405

[5] Kanzaki, M., Wachi, N. and Onuki, T. (2011) Simulating Video-Assisted Thoracoscopic Lung Resection Using a Virtual 3-Dimensional Pulmonary Model on a Personal Computer. The Journal of Thoracic and Cardiovascular Surgery, 142, 243-244. http://dx.doi.org/10.1016/j.jtcvs.2011.02.044

[6] Fukuhara, K., Akashi, A., Nakane, S. and Tomita, E. (2008) Preoperative Assessment of the Pulmonary Artery by Three-Dimensional Computed Tomography before Video-Assisted Thoracic Surgery Lobecotomy. European Journal of Cardiothoracic Surgery, 34, 875-877. http://dx.doi.org/10.1016/j.ejcts.2008.07.014

[7] Masuda, Y., Marutsuka, T. and Saishoji, T. (2013) Lung Resection for Lung Cancer in Patient with Situs Inversus Totalis. Kyobu Geka, 66, 481-484.

[8] Wojcik, J., Grodzki, T., Bielewicz, M., Wojtys, M., Kubisa, B., Pierog, J. and Wojcik, N. (2013) Lung Cancer in Situs Inversus Totalis (SIT)-Literature Review. Advances in Medical Sciences, 58, 1-8. http://dx.doi.org/10.2478/v10039-012-0083-X

[9] Lee, E.Y., Boiselle, P.M. and Shamberger, R.C. (2010) Multidetector Computed Tomography and 3-Dimentional Imaging: Preoperative Evaluation of Thoracic Vascular and Tracheobronchial Anomalies and Abnormalities in Paediatric Patients. Journal of Paediatric Surgery, 45, 811-821. http://dx.doi.org/10.1016/j.jpedsurg.2009.12.013 